\title{
Une typologie des typologies des applications des TIC en éducation
}

\author{
Josianne Basque ${ }^{*}$ — Karin Lundgren-Cayrol** \\ Centre de recherche LICEF \\ Télé-université \\ 4750, avenue Henri-Julien, bureau 100 \\ Montréal, Q., Canada H2T 3E4 \\ * jbasque@teluq.uquebec.ca \\ **klundgre@licef.teluq.uquebec.ca
}

RÉSUMÉ. Cet article présente 29 typologies des applications éducatives des technologies de l'information et de la communication (TIC) proposées par divers auteurs depuis le début des années 1980. Trois grandes catégories de typologies sont distinguées. Les premières s'appuient sur des critères de classification reliés à l'acte d'enseignement/apprentissage (rôle pédagogique de l'ordinateur, degré d'autonomie de l'apprenant, type de stratégies pédagogiques ou étapes du processus d'enseignement). Les deuxièmes associent les usages des TIC aux divers types d'activités se déroulant dans un établissement d'enseignement ou aux acteurs y oeuvrant. Les dernières regroupent les applications des TIC en fonction de différentes caractéristiques cognitives d'un apprenant (impulsions à apprendre, capacités étendues ou restructurées par l'usage des TIC, étapes du processus d'apprentissage). L'analyse révèle une tendance à une spécialisation des typologies à certains contextes éducatifs, à certaines technologies ou à certaines approches théoriques. Les typologies gagnent en sophistication et s'intéressent de plus en plus au fonctionnement cognitif de l'apprenant.

ABSTRACT. This article reviews 29 typologies of ICT (information and communication technologies) uses in education proposed since 1980 by researchers and professionals in the field. Three typology categories are distinguished: teaching-learning centered, schoolcentered and learner-centered. The first use as classification criteria: the roles attributed to a computer, the level of learner control and the instructional strategies or phases. The second category classifies ICT uses by type of school activities or type of actor in a school. The last group classifies ICT uses according to the cognitive functions supported by ICTs or according to some learning or information-processing phases. Typologies appear to become more specialized to express the use of a single technology, a learning context or a theoretical approach. They also become more sophisticated and more predisposed to articulate the learner's cognitive functions.

MOTS-CLÉS: Technologies éducatives, Applications pédagogiques des TIC, Classification, Typologies.

KEYWORDS: Educational technologies, Educational uses of ICT, Classification, Typologies.

Sciences et techniques éducatives. Volume $9-n^{\circ} 3-4 / 2002$, pages 263-298. 


\section{Introduction}

Au cours des dernières décennies, une quantité phénoménale de matériels, de logiciels et de services s'appuyant sur l'informatique, la microélectronique, les télécommunications (notamment les réseaux), le multimédia et l'audiovisuel de toutes sortes ont été développés. On les regroupe aujourd'hui généralement sous le vocable de technologies de l'information et de la communication (TIC). Ces technologies, lorsqu'elles sont combinées et interconnectées, permettent de rechercher, de stocker, de traiter et de transmettre des informations sous forme de données de divers types (texte, son, images fixes, images animées, etc.) et permettent l'interactivité entre des personnes, et entre des personnes et des machines.

Au cours des années 1960 et 1970, les premières expériences d'utilisation de l'ordinateur étaient tentées en éducation, dans le prolongement des travaux sur les machines à enseigner (Pressey, 1926) et sur l'enseignement programmé (Skinner, 1954). Au fur et à mesure des développements technologiques, de la diffusion des technologies auprès du grand public ainsi que de l'émergence de diverses initiatives de pédagogues à travers le monde et de recherches en technologie de l'éducation, les usages des TIC à des fins pédagogiques se sont multipliés, autant dans les milieux formels qu'informels d'éducation. Il est donc rapidement apparu nécessaire de répertorier et de catégoriser ces différents usages afin d'offrir aux chercheurs et praticiens en éducation un cadre leur permettant de bien cerner les applications possibles des TIC dans leur domaine et de partager un vocabulaire commun pour les désigner. Pour ce faire, une catégorisation des TIC reposant sur leurs seules caractéristiques techniques ne se révélait guère utile; d'autres critères plus près du monde de l'éducation devaient être identifiés.

Depuis le début des années 1980, plusieurs auteurs ont donc proposé des typologies des applications éducatives de l'ordinateur, de l'informatique et, plus récemment, des TIC. Une typologie est un «système de description, de comparaison, de classification, voire d'interprétation ou d'explication des éléments d'un ensemble, à partir de critères jugés pertinents, qui permet de ramener d'une façon simplifiée à quelques types fondamentaux une multiplicité d'objets ou de phénomènes distinct » (Sauvé, 1992, dans Legendre, 1993, p. 1382). Bref, une typologie constitue une sorte de carte conceptuelle ${ }^{1}$ et, en ce sens, contribue significativement à faire avancer les connaissances dans ce domaine, comme le fut, en biologie, la célèbre typologie Systema naturae de Karl von Linné, proposée en 1735 .

Sur le plan théorique, le fait de mener une réflexion sur les critères les plus pertinents pour classifier des phénomènes contribue significativement au

1 Dans une carte conceptuelle typologique, les liens entre les concepts sont essentiellement des liens de spécialisation » (sorte de). 
développement d'une discipline. Comme le souligne Seels (1997), les classifications résument une grande quantité de connaissances et permettent de faire des prédictions et des comparaisons auxquelles personne n'aurait pensé sans elles. Une typologie sert à réduire la diversité des éléments d'un ensemble en quelques types plus signifiants et, ainsi, à réduire la complexité d'un phénomène. Ce faisant, une typologie peut exercer une fonction descriptive (elle nous permet de mesurer le degré de déviation d'un cas réel par rapport au type de référence), une fonction interprétative (elle nous permet de révéler le ou les principes organisateurs d'une réalité et (ou) une fonction prédictive (elle nous permet de prédire le comportement d'un objet appartenant à un type particulier et de prédire l'existence d'autres catégories) (Legendre, 1993).

Pour un pédagogue ou un chercheur, une typologie des applications des TIC en l'éducation peut donc s'avérer intéressante à plus d'un titre: elle peut servir à analyser un nouvel usage des TIC en éducation par une comparaison avec les autres catégories de la typologie, à mieux comprendre le phénomène dans son ensemble, à y jeter un regard neuf, à en apprécier la complexité et la richesse, à apparenter des usages qui, au premier abord, semblent très différents ou, au contraire, à en distinguer qui semblent d'emblée très semblables, à rechercher de nouveaux critères de classification afin de trouver des principes organisateurs reflétant des approches éducatives nouvelles, etc.

Toute classification dans un domaine exprime une certaine vision de ce domaine, à un moment donné de l'histoire de son développement. Faire un tour d'horizon des typologies des applications des technologies informatiques en éducation proposées à ce jour par différents chercheurs, penseurs ou praticiens du monde de l'éducation, nous est donc apparu un exercice intéressant. C'est ce que nous faisons dans cet article, en présentant vingt-neuf typologies proposées au cours des trente dernières années. Nous avons nous-mêmes fait un exercice de classification de ces typologies, ce qui nous permet de proposer une typologie des typologies des applications des TIC en éducation.

Bien que nous ne prétendions pas avoir repéré toutes les typologies existantes, l'échantillon présenté nous permet de dégager quelques tendances qui ont marqué l'histoire d'une discipline en pleine effervescence : la technologie éducative.

\section{Méthode de constitution du corpus}

Pour constituer notre corpus des typologies des applications des TIC en éducation, nous avons repéré une première série de documents dans nos collections documentaires personnelles cumulées au cours de nos vingt ans de pratique et de recherche dans le domaine de la technologie éducative. Nous avons ensuite effectué une recherche documentaire dans les banques ERIC et FRANÇIS ainsi que dans l'Internet au moyen des engins de recherche Google et Copernic, en utilisant divers mots-clés pour désigner chacun des concepts visés (Classification OU Typolog* 
OU Taxonom*; Applications OU Usages OU Utilisations; Technolog* OU TIC OU Ordinateur OU Informatique; Éducation OU Enseignement). La recherche documentaire a été effectuée en langue française et anglaise. L'informatique s'étant développée en éducation surtout à partir des années 80 , nous n'avons pas remonté au-delà de cette date. Enfin, un certain nombre de typologies ont été repérées parce qu'elles étaient signalées dans les documents déjà sélectionnés.

Pour être retenu, un document devait proposer des catégories d'applications de l'ordinateur ou des TIC en contexte éducatif, et ce, en fonction d'une ou plusieurs dimensions touchant l'éducation. Les classifications fondées uniquement sur les caractéristiques techniques des TIC, sur la nature des informations qu'elles permettent de traiter (texte, image, etc.) et (ou) les processus qu'elles permettent d'appliquer sur ces informations (ex. composition graphique, traitement de texte, communication, etc.) ont été rejetées. En bref, la classification proposée devait avoir un lien quelconque avec le monde éducatif.

Le terme application est pris ici dans un sens assez large. Certains auteurs tels Baron et Bruillard (1996) font une distinction entre les usages et les utilisations de l'informatique en éducation. Les usages désignent les utilisations observées en situation éducative et encadrées par des formateurs alors que les utilisations sont celles qui sont prévues ou prescrites par les concepteurs des outils informatiques. Cette distinction n'est pas faite explicitement par les auteurs des typologies recensées ; nous n'en avons donc pas tenu compte. Dans les pages qui suivent, nous utilisons indifféremment les termes usages, utilisations et applications des TIC en éducation.

\section{Vue d'ensemble des typologies recensées}

Le tableau 1 présente les vingt-neuf typologies recensées dans l'ordre chronologique de leur publication. Pour chacune, le pays d'origine des auteurs, la dénomination utilisée par ceux-ci pour délimiter l'univers faisant l'objet de la classification et le critère principal de classification utilisé sont spécifiés. Ce dernier n'étant pas toujours explicite, nous l'avons inféré dans certains cas, en examinant les catégories proposées. Ces cas sont identifiés par un astérisque dans le tableau 1.

\subsection{Les auteurs et l'année de publication des typologies}

Parmi les typologies recensées, la plus ancienne, et probablement la plus connue, a été proposée par Taylor en 1980; onze autres ont été produites dans les années 1980. Douze typologies datent des années 1990 et trois ont été proposées depuis le début du millénaire. Notons qu'aucune date de publication n'est indiquée pour deux typologies trouvées sur la toile, soit celle de Denis et celle du ministère français de la Jeunesse, de l'Éducation nationale et de la Recherche (MJENR). 


\begin{tabular}{|c|c|c|c|}
\hline Auteurs & Pays & $\begin{array}{c}\text { Dénomination de } \\
\text { l'univers visé }\end{array}$ & $\begin{array}{c}\text { Critère principal de } \\
\text { classification }\end{array}$ \\
\hline 1. Taylor (1980) & États-unis & $\begin{array}{l}\text { Modes d'usages de } \\
\text { l'informatique en } \\
\text { éducation }\end{array}$ & $\begin{array}{l}\text { * Rôle de l'ordinateur } \\
\text { auprès de l'apprenant }\end{array}$ \\
\hline 2. Watts (1981) & Canada & $\begin{array}{l}\text { Usages de l'ordinateur } \\
\text { en éducation }\end{array}$ & $\begin{array}{l}\text { * Ensemble des activités } \\
\text { d'une école que les TIC } \\
\text { peuvent supporter }\end{array}$ \\
\hline 3. Roecks (1981) & États-Unis & $\begin{array}{l}\text { Usages de l'ordinateur } \\
\text { en éducation }\end{array}$ & $\begin{array}{l}\text { * Ensemble des activités } \\
\text { d'une école que les TIC } \\
\text { peuvent supporter }\end{array}$ \\
\hline 4. Dubuc (1982) & Canada & $\begin{array}{l}\text { Applications } \\
\text { pédagogiques de } \\
\text { l'ordinateur }\end{array}$ & $\begin{array}{l}\text { Principaux acteurs du } \\
\text { système d'éducation } \\
\text { concernés par l'utilisation } \\
\text { de l'ordinateur }\end{array}$ \\
\hline $\begin{array}{l}\text { 5. Schultz et Hart } \\
(1983)\end{array}$ & États-unis & $\begin{array}{l}\text { Vue d'ensemble des } \\
\text { ordinateurs en } \\
\text { éducation }\end{array}$ & $\begin{array}{l}\text { *Ensemble des usages } \\
\text { possibles en éducation }\end{array}$ \\
\hline 6. Plante (1984) & Canada & $\begin{array}{l}\text { Applications } \\
\text { pédagogiques de } \\
\text { l'ordinateur }\end{array}$ & $\begin{array}{l}\text { Degré de contrôle de } \\
\text { l'ordinateur sur l'activité } \\
\text { de l'apprenant }\end{array}$ \\
\hline 7. Aylwin (1984) & Canada & $\begin{array}{l}\text { Utilisations de } \\
\text { l'informatique dans un } \\
\text { établissement scolaire }\end{array}$ & $\begin{array}{l}\text { * Ensemble des activités } \\
\text { d'une école que les TIC } \\
\text { peuvent supporter }\end{array}$ \\
\hline 8. Sauvé (1984) & Canada & $\begin{array}{l}\text { Applications } \\
\text { pédagogiques de } \\
\text { l'ordinateur }\end{array}$ & $\begin{array}{l}\text { * Rôle de l'ordinateur } \\
\text { dans la relation } \\
\text { pédagogique }\end{array}$ \\
\hline 9. Bork (1984) & États-unis & $\begin{array}{l}\text { Modes d'usage de } \\
\text { l'ordinateur en } \\
\text { éducation }\end{array}$ & $\begin{array}{l}\text { * Rôle de l'ordinateur } \\
\text { dans la relation } \\
\text { pédagogique }\end{array}$ \\
\hline $\begin{array}{l}\text { 10. Thomas et } \\
\text { Boysen (1984) }\end{array}$ & États-unis & $\begin{array}{l}\text { Usages éducatifs de } \\
\text { l'ordinateur }\end{array}$ & $\begin{array}{l}\text { Étapes du processus } \\
\text { d'apprentissage }\end{array}$ \\
\hline $\begin{array}{l}\text { 11. Knezek, } \\
\text { Rachlin et } \\
\text { Scannell }\end{array}$ & États-unis & $\begin{array}{l}\text { Informatique } \\
\text { éducative }\end{array}$ & $\begin{array}{l}\text { * Ensemble des activités } \\
\text { d'une école que les TIC } \\
\text { peuvent supporter }\end{array}$ \\
\hline
\end{tabular}


6 Sciences et techniques éducatives. Volume $9-n^{\circ} 3-4 / 2002$, pages 263-298.

\begin{tabular}{|c|c|c|c|}
\hline Auteurs & Pays & $\begin{array}{c}\text { Dénomination de } \\
\text { l'univers visé }\end{array}$ & $\begin{array}{c}\text { Critère principal de } \\
\text { classification }\end{array}$ \\
\hline $\begin{array}{l}\text { 12. Alessi et } \\
\text { Trollip (1991) }\end{array}$ & États-unis & $\begin{array}{l}\text { Programmes } \\
\text { d'enseignement assisté } \\
\text { par ordinateur }\end{array}$ & $\begin{array}{l}\text { Étapes du processus } \\
\text { d'enseignement }\end{array}$ \\
\hline 13. Chacón (1992) & Vénézuela & $\begin{array}{l}\text { Médias informatiques } \\
\text { en éducation à } \\
\text { distance }\end{array}$ & $\begin{array}{l}\text { «Modes utilisateur» } \\
\text { (user-modes) et évolution } \\
\text { des technologies }\end{array}$ \\
\hline $\begin{array}{l}\text { 14. Paquette } \\
\text { (1993) }\end{array}$ & Canada & $\begin{array}{l}\text { Applications } \\
\text { pédagogiques de } \\
\text { l'ordinateur }\end{array}$ & $\begin{array}{l}\text { Stratégies, moyens } \\
\text { didactiques et types de } \\
\text { connaissances }\end{array}$ \\
\hline 15. Means (1994) & États-unis & $\begin{array}{l}\text { Technologies } \\
\text { éducatives }\end{array}$ & $\begin{array}{l}\text { Manières d'utiliser les } \\
\text { ordinateurs pour } \\
\text { l'enseignement }\end{array}$ \\
\hline $\begin{array}{l}\text { 16. Jonassen } \\
(1995)\end{array}$ & États-unis & $\begin{array}{l}\text { Technologies qui } \\
\text { supportent } \\
\text { l'apprentissage } \\
\text { significatif }\end{array}$ & $\begin{array}{l}\text { Rôle des technologies } \\
\text { dans un processus } \\
\text { d'apprentissage } \\
\text { significatif }\end{array}$ \\
\hline 17. Collis (1996) & Hollande & $\begin{array}{l}\text { Usages des ordinateurs } \\
\text { en éducation }\end{array}$ & $\begin{array}{l}\text { *Acteurs apprenant et } \\
\text { enseignant }\end{array}$ \\
\hline $\begin{array}{l}\text { 18. Bruce et } \\
\text { Levin (1997) }\end{array}$ & États-unis & $\begin{array}{l}\text { Usages des } \\
\text { technologies } \\
\text { éducatives, en se } \\
\text { centrant sur l'aspect } \\
\text { médiatique des } \\
\text { technologies }\end{array}$ & $\begin{array}{l}\text { Les quatre impulsions } \\
\text { naturelles des individus, } \\
\text { selon Dewey (1943) }\end{array}$ \\
\hline 19. Séguin (1997) & Canada & $\begin{array}{l}\text { Applications } \\
\text { pédagogiques } \\
\text { d'Internet }\end{array}$ & Activités d'apprentissage \\
\hline $\begin{array}{l}\text { 20. Iiyoshi et } \\
\text { Hannafin } \\
(1998)\end{array}$ & États-unis & $\begin{array}{l}\text { Outils cognitifs } \\
\text { d'environnements } \\
\text { ouverts d'appren- } \\
\text { tissage }\end{array}$ & $\begin{array}{l}\text { Modèle du processus de } \\
\text { traitement de } \\
\text { l'information }\end{array}$ \\
\hline $\begin{array}{l}\text { 21. Baumgartner } \\
\text { et Payr (1998) }\end{array}$ & Autriche & $\begin{array}{l}\text { Applications de } \\
\text { l'internet pour } \\
\text { l'apprentissage }\end{array}$ & $\begin{array}{l}\text { Modèle tridimensionnel } \\
\text { d'apprentissage }\end{array}$ \\
\hline
\end{tabular}




\begin{tabular}{|c|c|c|c|}
\hline Auteurs & Pays & $\begin{array}{c}\text { Dénomination de } \\
\text { l'univers visé }\end{array}$ & $\begin{array}{c}\text { Critère principal de } \\
\text { classification }\end{array}$ \\
\hline $\begin{array}{l}\text { 22. Basque et al., } \\
\text { (1998) }\end{array}$ & Canada & $\begin{array}{l}\text { Utilisations des TIC } \\
\text { dans une école typique }\end{array}$ & Processus d'une école \\
\hline 23. Forcier (1999) & États-unis & $\begin{array}{l}\text { Usages fonctionnels } \\
\text { de l'ordinateur en } \\
\text { éducation }\end{array}$ & * Processus d'une école \\
\hline 24. Limbos (1999) & Belgique & $\begin{array}{l}\text { Utilisations } \\
\text { pédagogiques de } \\
\text { l'ordinateur }\end{array}$ & Situations d'apprentissage \\
\hline $\begin{array}{l}\text { 25. Jonassen } \\
(2000)\end{array}$ & États-unis & $\begin{array}{l}\text { Applications } \\
\text { pédagogiques de } \\
\text { l'ordinateur }\end{array}$ & $\begin{array}{l}\text { Phases de l'histoire des } \\
\text { usages pédagogiques de } \\
\text { l'ordinateur et habiletés } \\
\text { cognitives supportées par } \\
\text { les TIC }\end{array}$ \\
\hline $\begin{array}{l}\text { 26. De Vries } \\
\text { (2001) }\end{array}$ & France & $\begin{array}{l}\text { Logiciels } \\
\text { d'apprentissage }\end{array}$ & $\begin{array}{l}\text { Fonction pédagogique } \\
\text { visée par les enseignants } \\
\text { ou les concepteurs }\end{array}$ \\
\hline 27. Lebrun (2002) & Belgique & $\begin{array}{l}\text { Supports } \\
\text { technologiques }\end{array}$ & $\begin{array}{l}\text { Modalités de dispositifs } \\
\text { pédagogiques }\end{array}$ \\
\hline 28. Denis (http) & Belgique & $\begin{array}{l}\text { Utilisations } \\
\text { pédagogiques de } \\
\text { l'ordinateur }\end{array}$ & Objectifs poursuivis \\
\hline $\begin{array}{l}\text { 29. Ministère de la } \\
\text { Jeunesse, de } \\
\text { l'Éducation } \\
\text { nationale et de } \\
\text { la Recherche } \\
\text { (http) }\end{array}$ & France & $\begin{array}{l}\text { Usages pédagogiques } \\
\text { des TIC pour les } \\
\text { élèves et les } \\
\text { enseignants }\end{array}$ & $\begin{array}{l}\text { * Processus effectués par } \\
\text { l'enseignant et }(\mathrm{ou}) \\
\text { l'élève }\end{array}$ \\
\hline
\end{tabular}

* Critère non explicité par les auteurs de la typologie

Tableau 1. Les 29 typologies des applications des TIC en éducation selon l'ordre chronologique de leur publication 
Treize typologies proviennent d'auteurs américains et huit d'auteurs canadiens, dont sept sont du Québec. Enfin, sept nous viennent d'Europe et une autre d'un chercheur sud-américain.

Les typologies sont proposées majoritairement par des chercheurs universitaires et quelques-unes le sont par des professionnels œuvrant dans le monde de l'éducation. Elles sont diffusées, pour la plupart, dans des revues et ouvrages spécialisés en éducation et en technologie éducative. Certaines typologies ont toutefois donné lieu à une diffusion plus restreinte : c'est le cas de deux typologies proposées dans des documents ayant fait partie du matériel de certains cours de la Télé-université (Sauvé, 1984; Paquette, 1993). Quatre autres, soit celles de Séguin (1997), de Basque et al. (1998), de Denis (http), du MJENR (http), sont diffusées uniquement, à notre connaissance, sur la toile.

\subsection{La dénomination utilisée pour délimiter l'univers faisant l'objet de la classification}

Les termes exacts - ou traduits, le cas échéant - utilisés par les auteurs pour délimiter l'univers faisant l'objet de la classification sont spécifiés dans la deuxième colonne du tableau 1. Un coup d'œil à ces dénominations nous permet de constater que le vocabulaire a évolué pour faire référence au phénomène des TIC en éducation. Jusqu'au milieu des années 90 , on parle d'applications - ou d'usages ou d'utilisations - de l'ordinateur ou de l'informatique en éducation ou à l'école. En français, l'acronyme APO, pour applications pédagogiques de l'ordinateur, était alors largement utilisé. Par la suite, le terme technologies et l'acronyme TIC commencent à apparaître à la place de ceux d'ordinateur et d'informatique ${ }^{2}$. Quant au terme média, qui était largement utilisé dans les milieux éducatifs dans les années soixante et soixante-dix pour désigner les supports technologiques ou les technologies utilisées à des fins d'enseignement et d'apprentissage, il n'est utilisé que par Chacón (1992), plus spécifiquement dans la formule «computer media». Plusieurs définitions du terme média semblent faire référence exclusivement aux supports technologiques et aux technologies qui servent à véhiculer de l'information (voir, par exemple, Baumgartner et Payr, 1998 ; Heinich et al., 1993 ; Reiser, 2001; Romizowski, 1992). Chacón semble de cet avis lorsqu'il définit ce qu'il entend par «computer media»: «hardware and software for conveying or transmitting something to the user » (p. 13). Toutefois, en examinant la typologie de Chacón, on conclut que ce dernier parle bien ici des TIC, telles qu'elle sont définies au début de cet article. Cela inclut non seulement les technologies servant à transmettre de l'information mais également à la rechercher, à la stocker, à la traiter et à la communiquer.

2 Ce qui a fait naître, au cours des dernières années, l'acronyme APTIC (applications pédagogiques des TIC). 
Bruce et Levin (1997) qualifient, pour leur part, leur vision des technologies éducatives de «médiatique »; leur approche du média se démarque de la conception restreinte du média en tant que véhicule d'informations. Pour eux, le terme média met surtout en évidence la fonction de médiation des technologies: celles-ci permettent de relier les étudiants aux autres apprenants, aux enseignants, aux autres technologies, aux idées et au monde physique.

Par ailleurs, nous observons une spécialisation de plus en plus marquée des typologies à certains contextes éducatifs, à certaines technologies ou même à certaines approches théoriques. Par exemple, Chacón (1992) se concentre sur les applications des technologies en formation à distance - bien qu'il prétende que la typologie pourrait tout aussi bien convenir à l' « éducation traditionnelle»-, alors que Séguin (1997) et Baumgartner et Payr (1998) traitent des usages pédagogiques d'Internet. Iiyoshi et Hannafin (1998) se concentrent, pour leur part, sur les «environnements ouverts d'apprentissage » (open-ended learning environments), précisant que ce vocable désigne des environnements qui donnent à l'apprenant la responsabilité d'accéder, d'organiser et d'analyser l'information fournie. Quant à Jonassen (1995), il adopte la perspective des technologies qui supportent une orientation pédagogique particulière, soit celle de l'«apprentissage significatif $»^{3}$. Cette tendance à la spécialisation des typologies témoigne du développement du domaine des TIC appliquées à l'éducation, tant sur le plan théorique que sur le plan pratique.

\subsection{Les critères de classification}

Notre analyse nous a amenées à faire les constats généraux suivants, relativement aux critères de classification utilisés par les auteurs des typologies et aux catégories qui en découlent :

- Certains auteurs utilisent plus d'un critère de classification pour élaborer leur typologie. Dans ces cas, les typologies sont souvent accompagnées de représentations graphiques (notamment la forme cubique) pour illustrer leurs principes organisateurs.

- Il n’y a généralement pas de relation hiérarchique entre les catégories des classifications proposées par les auteurs. En ce sens, il s'agit véritablement de typologies et non de taxonomies, deux termes souvent confondus à tort. Comme l'explique Sauvé (1992, dans Legendre, 1993), une taxonomie est un système de classification systématique et hiérarchisé. Pour donner un exemple d'une taxonomie en éducation, citons la taxonomie des objectifs pédagogiques de Bloom, dans laquelle ceux-ci sont d'abord classifiés selon les domaines cognitif, affectif et psychomoteur, puis, à l'intérieur de chaque domaine, selon un ordre hiérarchisé de

3 Pour Jonassen, un apprentissage significatif est actif, constructif, collaboratif, intentionnel, conversationnel, contextualisé et réflexif. 
processus de pensée de plus en plus complexes (Connaissance, Compréhension, Application, Analyse, Synthèse et Évaluation). Une typologie n'implique pas d'emblée de hiérarchie; elle ne fait que rassembler «plusieurs caractéristiques en un ensemble organisé, une macrocatégorie jugée signifiante » (Sauvé, 1992, dans Legendre, 1993).

- Certains auteurs présentent leur typologie comme un ensemble fermé de catégories d'applications pédagogiques des TIC, alors que d'autres auteurs se contentent d'identifier des catégories générales et présentent, pour chacune, des ensembles ouverts $d^{\prime}$ ' «exemples » d'usages représentatifs de cette catégorie. Cette dernière façon de faire se veut notamment plus sensible à l'évolution technologique et lève l'obligation d'exhaustivité, qui est, de toute manière, quasi impossible à respecter, compte tenu de l'ampleur des usages qui se sont rapidement étendus en éducation et en formation.

- Certains auteurs insistent pour dire que les catégories de leur typologie ne sont pas mutuellement exclusives. Assez souvent, une même TIC peut servir à plusieurs classes d'applications. La tendance à l'intégration des technologies et le mariage de multiples fonctionnalités dans des environnements technologiques de plus en plus complexes et étendus font en sorte que les efforts de classification deviennent de plus en plus difficiles, chaque TIC permettant de réaliser une diversité d'activités, autrefois exécutées à l'aide de programmes et équipements distincts.

- Tel que déjà mentionné, bon nombre d'auteurs (onze) ne spécifient pas le ou les critères qu'ils ont employés pour développer leur typologie. La plupart du temps, les auteurs ne donnent aucune information sur la méthodologie qu'ils ont utilisée pour développer leur typologie. À l'analyse, il nous a semblé évident qu'un certain nombre d'entre eux ont procédé de manière plutôt inductive, en partant d'une liste d'usages observés et en recherchant leurs points de convergence et de divergence. D'autres paraissent avoir procédé à l'inverse : à partir d'un modèle théorique, tel un modèle d'enseignement ou d'apprentissage, ils recherchent des usages des TIC qui peuvent s'intégrer aux dimensions du modèle. Ceux-là tendent à présenter longuement les catégories et à n'offrir qu'un ou deux exemples pour les illustrer, alors que les méthodes inductives conduiraient à des typologies plus extensives.

\section{Une typologie des typologies des applications des TIC en éducation}

Comme on peut le voir au tableau 2, nous proposons un regroupement des typologies des usages des TIC en éducation en trois catégories :

- Les typologies centrées sur l'acte d'enseignement/apprentissage. Ces typologies ont pour critère de classification une ou plusieurs variables définissant 


\begin{tabular}{|c|c|c|}
\hline $\begin{array}{c}\text { Classes } \\
\text { principales }\end{array}$ & Sous-classes & Typologies \\
\hline \multirow[t]{4}{*}{$\begin{array}{l}\text { Typologies } \\
\text { centrées sur l'acte } \\
\text { d'enseignement/ } \\
\text { apprentissage }\end{array}$} & $\begin{array}{l}\text { Rôle de l'ordinateur au sein } \\
\text { de la relation pédagogique }\end{array}$ & $\begin{array}{l}\text { Taylor }(1980) \\
\text { Sauvé }(1984) \\
\text { Bork }(1985) \\
\text { Means }(1994) \\
\text { Jonassen }(1995)\end{array}$ \\
\hline & $\begin{array}{l}\text { Degré d'autonomie de } \\
\text { l'apprenant }\end{array}$ & $\begin{array}{l}\text { Plante (1984) } \\
\text { Lebrun (2002) }\end{array}$ \\
\hline & $\begin{array}{l}\text { Types d'activités } \\
\text { pédagogiques et (ou) de } \\
\text { connaissances visées }\end{array}$ & $\begin{array}{l}\text { Paquette (1993) } \\
\text { Séguin (1997) } \\
\text { Baumgartner et Payr (1998) } \\
\text { Limbos (1999) } \\
\text { De Vries (2001) } \\
\text { Denis (http) } \\
\text { MJENR (http) }\end{array}$ \\
\hline & $\begin{array}{l}\text { Étapes du processus } \\
\text { d'enseignement }\end{array}$ & Alessi et Trollip (1991) \\
\hline \multirow[t]{2}{*}{$\begin{array}{l}\text { Typologies } \\
\text { centrées sur l'école }\end{array}$} & Types d'activités d'une école & $\begin{array}{l}\text { Watts (1981) } \\
\text { Roecks (1981) } \\
\text { Schultz et Hart (1983) } \\
\text { Aylwin (1984) } \\
\text { Knezek, Rachlin et Scannell } \\
\text { (1988) } \\
\text { Basque et al. (1998) } \\
\text { Forcier (1999) }\end{array}$ \\
\hline & Acteurs d'une école & $\begin{array}{l}\text { Dubuc (1982) } \\
\text { Collis (1996) }\end{array}$ \\
\hline \multirow{3}{*}{$\begin{array}{l}\text { Typologies } \\
\text { centrées sur } \\
\text { l'apprenant }\end{array}$} & $\begin{array}{l}\text { Impulsions de l'individu à } \\
\text { apprendre }\end{array}$ & Bruce et Levin (1997) \\
\hline & $\begin{array}{l}\text { Fonctions cognitives que } \\
\text { l'ordinateur permet d'étendre } \\
\text { ou de restructurer }\end{array}$ & $\begin{array}{l}\text { Chacòn (1992) } \\
\text { Jonassen (2000) }\end{array}$ \\
\hline & $\begin{array}{l}\text { Étapes du processus } \\
\text { d'apprentissage ou de } \\
\text { traitement de l'information }\end{array}$ & $\begin{array}{l}\text { Thomas et Boysen (1984) } \\
\text { Iioshi et Hannafin (1998) }\end{array}$ \\
\hline
\end{tabular}

Tableau 2. Une typologie des typologies des applications des TIC en éducation 
12 Sciences et techniques éducatives. Volume $9-\mathrm{n}^{\circ}$ 3-4/2002, pages 263-298.

une situation pédagogique. Quatre sous-catégories sont identifiées: (1) les typologies s'intéressant au rôle du nouvel « acteur » ordinateur au sein de la relation pédagogique, (2) celles qui mettent l'accent sur le degré de contrôle exercé par l'apprenant par rapport à celui qu'exerce l'ordinateur, (3) celles qui regroupent les usages des TIC selon les stratégies ou activités pédagogiques et (ou) les types de connaissances que les TIC permettent de supporter et (4) celles qui associent les TIC à des étapes spécifiques du processus d'enseignement.

- Les typologies centrées sur l'école. Ces typologies visent à répertorier l'ensemble des usages possibles des TIC au sein d'un établissement d'enseignement. Deux sous-catégories sont proposées : (1) les typologies qui associent les usages des TIC aux activités d'une école et (2) celles qui les associent plutôt aux acteurs de l'éducation.

- Les typologies centrées sur l'apprenant. Il s'agit de typologies dont le système de classification repose sur les différentes façons dont les TIC peuvent supporter les activités de l'apprenant. Trois sous-catégories sont proposées: (1) celles qui classifient les usages des TIC selon les «impulsions de l'apprenant », (2) celles qui les classifient selon les fonctions cognitives supportées par les technologies et (3) celles qui les classifient selon les étapes du processus d'apprentissage ou encore les étapes du processus humain de traitement de l'information.

La première catégorie est la plus fournie (quinze typologies), alors que la dernière catégorie en compte le moins (cinq). On dénombre, par ailleurs, neuf typologies centrées sur l'école. On retrouve des typologies récentes (parues depuis 1998) dans les trois catégories. Toutefois, elles sont légèrement plus nombreuses dans la catégorie des typologies centrées sur l'acte d'enseignement/apprentissage. Les paragraphes qui suivent décrivent plus en détail chacune des catégories de notre typologie.

\subsection{Les typologies centrées sur l'acte d'enseignement-apprentissage}

Un premier sous-groupe de typologies centrées sur l'acte d'enseignementapprentissage considèrent l'ordinateur comme un nouvel acteur au sein de la relation pédagogique. Ces typologies s'intéressent plus particulièrement au rôle spécifique adopté par ce nouvel acteur dans la relation pédagogique. C'est le cas de la typologie la plus ancienne que nous avons recensée (Taylor, 1980). Dans cette typologie des «modes d'usage de l'informatique en éducation », trois rôles sont identifiés pour l'ordinateur, selon la célèbre formule «Tutor, Tool, Tutee ».

Le premier rôle fait référence aux tutoriels. Ce rôle est décrit par Taylor de la manière suivante: "The computer presents some subject material, the student responds, the computer evaluates the response, and, from the results of the evaluation, determines what to present next. » (p. 3) L'ordinateur assume ici le rôle traditionnel de l'enseignant auprès de l'apprenant. Le deuxième rôle fait référence 
aux usages des divers logiciels-outils ou outils de productivité, encore peu nombreux à l'époque, permettant de produire et d'éditer des textes, d'exécuter des calculs, de faire des analyses statistiques, de produire des illustrations, etc. Le dernier rôle, celui de l'ordinateur-apprenant, désigne les situations où l'apprenanthumain prend la place de l'enseignant et utilise un langage de programmation pour « converser» avec l'ordinateur-apprenant. Par sa simplicité, sa formule-choc (les 3 « $\mathrm{T} »)$ et sa référence directe à la place de l'ordinateur dans l'acte pédagogique, cette typologie a été largement utilisée par les spécialistes du domaine.

Cette centration sur le rôle joué par l' « acteur » ordinateur au sein de la relation pédagogique est également le point de vue adopté par Sauvé (1984), par Bork (1985), par Jonassen (1995) et en partie par Means (1994). La première typologie reprend essentiellement les catégories de Taylor, bien que nommées différemment, soit Ordinateur, moyen d'enseignement (ordinateur-tuteur), Ordinateur, moyen d'apprentissage (ordinateur apprenant), Ordinateur, outil d'enseignementapprentissage (ordinateur outil), auxquelles est rajoutée une quatrième catégorie dans laquelle l'ordinateur est plutôt mis au service de l'enseignant pour, par exemple, gérer le cheminement pédagogique des étudiants ou encore gérer une banque d'instruments de mesure des apprentissages : c'est l'Ordinateur, instrument de gestion pédagogique.

La typologie de Bork (1985) est très semblable à cette dernière, bien qu'une catégorie associée à l'ordinateur en tant qu'objet de connaissance (ce que l'on nomme en anglais la computer literacy) y soit ajoutée.

Means (1994) utilise également le critère du rôle des technologies auprès de l'apprenant pour deux catégories de sa typologie, soit les technologies utilisées comme tuteur et les technologies utilisées comme outil. Elle semble toutefois s'appuyer sur un nouveau critère pour désigner ses deux autres catégories, soit les technologies utilisées pour explorer (ex. : laboratoires informatisés, micromondes, simulations, etc.) et les technologies utilisées pour communiquer (réseaux locaux, apprentissage interactif à distance, etc.). Dans ces deux derniers cas, l'auteure fait moins référence au rôle des technologies dans la relation pédagogique qu'aux activités de l'apprenant qu'elles soutiennent. Comme on le verra plus loin, ce dernier critère est utilisé par nombre d'autres auteurs : nous avons regroupé ces typologies sous la rubrique des « typologies centrées sur l'apprenant ».

Enfin, Jonassen (1995) offre une vision quelque peu différente du rôle joué par les technologies au sein de la relation pédagogique. Selon lui, les technologies peuvent avoir une fonction d'outils de productivité (traitement de texte, tableurs, édition électronique, édition graphique, etc.), une fonction de partenaires intellectuels (elles sont alors nommées mindtools) (bases de données, réseaux sémantiques, systèmes experts, conférence informatique, construction de multimédia et hypermédia, etc.) et enfin une fonction de contexte d'apprentissage permettant à l'apprenant de résoudre des problèmes et de construire ses connaissances (environnements d'apprentissage fondés sur les cas, environnements 
d'apprentissage intentionnels, hypertextes, micromondes, etc.). Cet auteur écarte d'emblée les utilisations de l'ordinateur où la technologie sert de véhicule d'informations ou de contrôleurs de l'enseignement : "Rather, they should be used as facilitators of thinking and knowledge construction» (p. 62) Nous verrons plus loin que Jonassen propose, en 2000, une version plus raffinée de cette typologie, dans laquelle il regroupe les outils cognitifs (mindtools) selon les diverses fonctions cognitives qu'ils permettent non seulement d'amplifier mais également de restructurer.

Plante (1984) s'intéresse également à la fonction de l'ordinateur dans la relation pédagogique, mais plutôt sous l'angle du degré de contrôle que l'apprenant exerce sur celui-ci et vice versa. Il situe les applications les plus courantes de l'époque sur un continuum allant de l'Ordinateur-contrôler/Étudiant contrôlé à l'Ordinateuroutil/Étudiant maître. Les didacticiels associés à l'enseignement assisté par ordinateur (E.A.O.), soit les exercices de répétition, les tutoriels, les jeux et les simulations se situent à la première extrémité du continuum. Les langages de programmation tels que Smalltalk, Logo, Pascal et Basic se situent à l'autre extrémité. Les progiciels (traitement de texte, banques de données, bases de données, logiciels de création musicale, etc.) sont placés au centre. Quant aux systèmes-auteurs et langages-auteurs, très populaires à l'époque, Plante postule qu'il est possible de les situer n'importe où sur le continuum.

Lebrun (2002) adopte une approche semblable pour classifier les «supports technologiques » utilisés en situation pédagogique. Il identifie trois «modalités de dispositifs pédagogiques ». Le mode réactif est celui où l'ordinateur a l'initiative en tant que source du savoir; c'est le cas, par exemple, lorsque l'enseignant utilise l'ordinateur comme tableau noir ou lorsque l'élève utilise un didacticiel. Le mode proactif est celui où l'apprenant a l'initiative et l'ordinateur répond à ses sollicitations, comme lorsque l'apprenant utilise un logiciel de simulation. Dans le mode mutuel et interpersonnel, l'apprenant et l'ordinateur ont tour à tour l'initiative; Lebrun fournit ici l'exemple des environnements immersifs pour illustrer cette modalité.

Parmi les typologies centrées sur l'acte d'enseignement-apprentissage, on retrouve un troisième sous-groupe de typologies, qui prennent les types d'activités ou de stratégies pédagogiques que les TIC permettent de supporter et (ou) les types de connaissances visées lors de leur utilisation comme critères de classification des utilisations éducatives des TIC. C'est le cas de Séguin (1997), qui s'appuie luimême en partie sur une typologie de Harris (1995) pour répertorier trentetrois applications pédagogiques de l'Internet, regroupées en six grandes catégories d'activités pédagogiques, appelées «modèles»: La communication interpersonnelle, La cueillette de données, Les travaux d'équipe, L'utilisation des ressources, La publication sur Internet et L'autoapprentissage. Plusieurs exemples d'activités expérimentées dans des classes réelles sont fournis pour chaque catégorie. 
Dans le même ordre d'idées, Limbos (1999) propose cinq situations d'apprentissage dans lesquelles les TIC peuvent être utilisées.: (1) Production d'écrits et ses exploitations (traitement de texte, le courrier électronique, les conversations électroniques, etc.), (2) Représentations mentales/Articulations des connaissances individuelles et collectives (création d'une page personnelle d'un site web ou d'un document hypertexte), (3) Résolution de problèmes / Réalisation de projets personnel (robots de plancher, tortue d'écran, robotique pédagogique, logiciels d'expérimentation et de simulation), (4) Consultation de (res)sources de références (cédéroms encyclopédiques, navigation dans Internet et (5) Acquisition de connaissances spécifiques et gestion de la construction de ces connaissances (didacticiels).

À l'instar de Séguin (1997), Baumgartner et Payr (1998) proposent une typologie des usages de l'Internet à des fins éducatives, mais ceux-ci adoptent un point de vue plus théorique sur la question. Ces auteurs s'appuient sur leur « modèle heuristique d'apprentissage » qui conjugue trois dimensions (représentées au moyen d'un cube) : la dimension de l'action de l'apprenant (allant de la perception et la mémorisation à l'invention, la coopération et la maîtrise), la dimension de l'enseignement/apprentissage $e^{4}$ (allant des faits aux situations complexes, en passant par les règles, la résolution de problèmes et la reconnaissance de formes) et la dimension de l'organisation $d u$ processus d'apprentissage (enseignement/explication, observation/aide support/coopération). La forme cubique est utilisée pour représenter graphiquement leur modèle. Les classes de leur typologie se trouvent au croisement de certains attributs des deux premières variables de ce modèle. Par exemple, au croisement de la dimension enseignement/apprentissage de type «faits et règles » et des actions de l'apprenant de type «mémoriser/recevoir», se trouvent la navigation dans des sites qui se limitent à présenter des informations; au croisement de la dimension enseignement/apprentissage « résoudre des problèmes » et des actions « décider/ sélectionner », se trouvent la navigation dans des sites présentant des problèmes à résoudre aux étudiants, etc.

Paquette (1993) utilise également la représentation cubique pour illustrer sa classification des applications pédagogiques de l'ordinateur, qui comprend trois dimensions : la dimension des stratégies, la dimension des moyens didactiques et la dimension des types de connaissances visées. Dans l'axe moyens didactiques, on retrouve cinq types de logiciels : exerciseurs, présentiels, simulateurs, progiciels et logiciels-outils à base de connaissances/systèmes tutoriels intelligents. L'axe des stratégies inclut les catégories suivantes : implantation et mémorisation; instruction systématique; analogie et transfert; expérimentation et induction; construction et découverte. Dans l'axe des connaissances, on retrouve les catégories suivantes : faits et données; procédures et savoir-faire; concepts et savoirs; structures et

4 En prenant connaissance des catégories définissant cette variable, il nous apparaît qu'il s'agit, en fait, des types de connaissances et d'habiletés visées dans la situation pédagogique. 
modèles; métaconnaissances. Les logiciels ne sont liés à aucune catégorie précise faisant partie des deux autres dimensions. Il revient à chaque usager de la typologie de faire cette analyse. En ce sens, il nous semble que nous n'avons pas affaire ici à une véritable classification (bien que l'auteur la qualifie ainsi). Le modèle ne manque cependant pas d'intérêt et nous invite à réfléchir à une autre façon d'envisager la réflexion sur les usages des TIC : plutôt que de les associer à des catégories fermées (ce qui conduit toujours à des cas limites ou encore à des cas pouvant être classés dans plusieurs catégories), peut-être faut-il répertorier les principales dimensions qui caractérisent les usages des TIC (types de connaissances visées, types de stratégies pédagogiques, etc.) et identifier, pour chaque dimension, une liste d'attributs possibles? Chaque cas d'utilisation des TIC serait alors analysé en fonction de cette grille.

De Vries (2001) classifie les logiciels d'apprentissage selon les fonctions pédagogiques qui leur sont attribuées par leurs concepteurs ou par les enseignants. À ces fonctions pédagogiques, elle associe non seulement un type spécifique de logiciel, mais également le point de vue théorique sous-jacent face à l'enseignement/apprentissage (cognitiviste, béhavioriste, constructiviste ou cognition située), la tâche généralement proposée aux élèves (lire, faire des exercices, dialoguer, jouer, etc.) et le statut accordé aux connaissances (présentation ordonnée, association, représentation, etc.). Huit fonctions pédagogiques sont définies: (1) Présenter de l'information (tutoriel), (2) Dispenser des exercices (exercices répétés), (3) Véritablement enseigner (tuteur intelligent; (4) Captiver l'attention et la motivation de l'élève (jeu éducatif); (5) Fournir un espace d'exploration (hypermédia); (6) Fournir un environnement pour la découverte de lois naturelles (simulation); (7) Fournir un environnement pour la découverte de domaines abstraits (micro-monde) et (8) Fournir un espace d'échange entre élèves (outils d'apprentissage collaboratif).

La typologie de Denis (http) peut également être classée parmi les typologies utilisant les types d'activités ou stratégies pédagogiques comme critère de classification, bien que l'auteure le définit comme étant les « objectifs poursuivis » dans les situations d'apprentissage basés sur des utilisations pédagogiques de l'ordinateur. Ceux-ci vont de l'enseignement/acquisition de notions ou de démarches (imagiciels, simulations, expérimentations assistées par ordinateur, tutoriels, exerciseurs, modélisations ou systèmes experts) à la gestion de l'enseignement et de l'apprentissage, en passant par la recherche d'informations (bases de données, Internet, encyclopédies, dictionnaires), la création de programmes ou logiciels multimédias (micromondes Logo, robotique pédagogique, conception de pages web ou de produits multimédias), la production de travaux avec des logiciels outils (traitement de texte, tableur, logiciels de dessin...) et la communication/ collaboration (courrier électronique, forums, newsgroups, chat, vidéoconférence).

Nous pouvons situer également la typologie proposée sur le site EducNet du Ministère de la jeunesse, de l'éducation nationale et de la recherche en France 
(www.educnet.education.fr) dans la catégorie des typologies répertoriant les usages éducatifs des TIC selon le type d'activités ou stratégies pédagogiques qu'elles permettent de supporter. Cette typologie inclut, en effet, cinq groupes d'activités: (1) Coopérer, mutualiser, (2) Produire, créer, (3) Rechercher, se documenter, (4) Former, s'auto-former et (5) Animer, organiser, conduire. Dans chacun de ces groupes, sont spécifiés les champs d'applications possibles, la valeur ajoutée des TIC et quelques exemples de pratiques. Par exemple, pour la première catégorie, la correspondance scolaire est proposée comme l'un des champs d'application; la valeur ajoutée est définie comme étant «motiver l'expression écrite » et un exemple est fourni : « le Village imaginaire ».

Alessi et Trollip (1991) associent, pour leur part, les usages pédagogiques de l'ordinateur aux étapes du processus d'enseignement, justifiant ainsi l'ajout d'un quatrième sous-groupe dans la catégorie des typologies centrées sur l'acte d'enseignement/apprentissage. Leur modèle d'enseignement inclut quatre étapes : Présenter l'information, Guider l'étudiant, Faire faire des exercices à l'étudiant et Évaluer l'apprentissage. Selon eux, les tutoriels sont généralement utilisés aux deux premières étapes, les exerciseurs et jeux informatisés à la troisième étape et les tests informatisés à la dernière étape. Quant aux simulations, elles peuvent être utilisées à l'une ou l'autre étape. Ce modèle d'enseignement nous semble fortement associé à une approche béhavioriste de l'apprentissage et laisse peu de place à des approches plus constructivistes. Selon Bruce et Levin (1997), cette classification repose sur un modèle d'enseignement explicite, et, de ce fait, plusieurs TIC disponibles aujourd'hui ne trouveraient pas leur place dans cette typologie. C'est le cas d'un navigateur web, par exemple, puisqu'il ne suppose pas de contrôle sur le processus de présentation de l'information, que les interactions de l'étudiant avec le navigateur ne sont pas guidées, qu'il n'y a aucun exercice pour favoriser la rétention et qu'il n'y a pas d'évaluation explicite.

\subsection{Les typologies centrées sur l'école}

Dans les années 1980, plusieurs auteurs (Watts, 1981; Roecks, 1981; Dubuc, 1982; Aylwin, 1984) ont proposé des typologies qui situent les applications liées à l'acte pédagogique au sein d'un ensemble plus large d'utilisations possibles de la technologie informatique dans un établissement scolaire. Cette préoccupation n'est à peu près plus présente dans les années 1990, où l'on se concentre plus spécifiquement sur les TIC utilisées à des fins exclusives d'enseignement et d'apprentissage. Il est utile de rappeler ici que l'informatique a pénétré plus rapidement le secteur administratif que le secteur pédagogique de l'éducation. Il était donc sans doute nécessaire, à l'époque, de bien faire reconnaître la spécificité et l'importance des applications strictement pédagogiques dans l'ensemble des processus scolaires, ne serait-ce que pour justifier la nécessité d'investir des ressources pour la recherche et le développement dans ce secteur. Aujourd'hui, les TIC ont, semble-t-il, suffisamment droit de cité dans les classes pour que ce besoin 
de faire cette distinction entre l'administration scolaire et la pédagogie ne soit plus nécessaire. De plus, la notion même d'établissement scolaire en tant que lieu physique réel ayant perdu quelque peu de sa consistance avec la multiplication d'écoles et de campus virtuels, les applications pédagogiques des TIC ne sont plus aussi strictement vues comme se déroulant dans les lieux traditionnels d'enseignement.

Certains travaux récents prennent néanmoins l'ensemble des activités se déroulant dans une école comme point d'ancrage pour réfléchir aux usages éducatifs des TIC. Forcier (1999), par exemple, les regroupe en trois grandes catégories : les usages pour l'administration (budget, inventaire, dossiers des étudiants, etc.), les usages pour la recherche action (stockage de données, analyses statistiques) et les usages pour l'enseignement et l'apprentissage. Cette dernière catégorie inclut les usages axés sur l'enseignant (culture informatique, enseignement assisté par ordinateur, enseignement géré par ordinateur et conception de matériel pédagogique) ou ceux axés sur l'apprenant visant à soutenir la construction de ses connaissances (traitement de l'information, résolution de problèmes, apprentissage et conception multimédia).

Parmi les travaux récents, on retrouve également le «modèle adaptable d'une école informatisée » de Basque et al. (1998), qui propose une «vision systémique et adaptable des multiples usages possibles des TIC dans l'ensemble des processus d'une école typique» (p. 1). Le modèle identifie les quatre processus jugés fondamentaux d'une école (Enseigner, Apprendre, Gérer l'école et Assurer l'exploitation d'un centre de ressources multimédias). Chacun de ces processus est décomposé en sous-processus (18 au total), ceux-ci étant eux-mêmes décomposés en tâches (64 au total), et les acteurs habituels de chacun d'entre eux sont identifiés (apprenant, enseignant, gestionnaire, etc.). L'apport potentiel des TIC à la réalisation de chacun de ces processus, sous-processus et tâches, exécutés par divers acteurs de l'école y est décrit dans des fiches associées à l'ensemble de ces activités.

Ces travaux constituent, en quelque sorte, un approfondissement, une systématisation, un élargissement et une mise à jour des travaux amorcés dans les années 1980 par Watts (1981), Roecks (1981), Schultz et Hart (1983) et Aylwin (1984). Watts (1981) présente une liste beaucoup plus restreinte (une douzaine) d'activités scolaires et fournit, pour chacune, quelques exemples d'usages de l'informatique pouvant en faciliter l'exécution. Roecks (1981), à la suite d'une recension des usages de l'informatique souhaitées dans les cinquante écoles desservies par le centre de service éducatif où il travaille, procède à leur classification selon la typologie de Watts et en conclut que la plupart peuvent facilement être classées dans les douze catégories identifiées par ce dernier. Il en rajoute toutefois une treizième, intitulée Coordination institutionnelle, qui touche le partage d'informations et la coordination de services informatiques à l'intérieur d'un établissement ou inter établissements. Pour sa part, Aylwin (1984), qui travaille alors dans un établissement d'enseignement collégial du Québec, recense neuf types d'activités d'un établissement scolaire, allant de l'administration générale à 
l'enseignement de l'informatique, en passant par la documentation pédagogique, l'encadrement de l'enseignement, l'apprentissage, etc.; en outre, il identifie brièvement, pour chacune, des avantages obtenus par l'usage de l'informatique. Quant à Schultz et Hart (1983), ils distinguent deux grandes catégories d'utilisation des ordinateurs en éducation. Il y a, d'une part, les activités visant l'étude des ordinateurs par (1) le développement d'une culture informatique (computer awareness) (connaissance de l'histoire, des impacts des ordinateurs sur la société, du matériel et des logiciels) et (2) d'une compétence informatique (programmation et utilisation du matériel). Les auteurs regroupent, d'autre part, les applications de l'ordinateur en tant que véhicules: (1) l'enseignement (exerciseurs, tutoriels, simulations et logiciel de résolution de problèmes), (2) la gestion de données telles que les présences, la diplomation, les résultats scolaires, etc.) et la gestion de données relatives à l'enseignement (objectifs des programmes, planification, etc.) et (3) l'emmagasinage et la récupération d'informations (telles que des banques documentaires).

La perspective « école » est également adoptée par Knezek, Rachlin et Scannell (1988), qui développent un peu plus les catégories des auteurs précédents. On retrouve trois catégories principales : les applications visant le production du savoir (knowledge generation), celles visant la diffusion du savoir (knowledge dissemination) et celles visant la gestion de l'information (information management). Dans la catégorie diffusion du savoir, nous retrouvons les trois « $\mathrm{T}$ » de Taylor (1980) : Tutor, Tool, Tutee; à ceux-ci s'ajoute un quatrième « T», intitulé The computer as a topic (l'ordinateur, objet d'apprentissage), qui correspond à ce que d'autres appellent la computer literacy. Dans la catégorie "production du savoir ", on reconnait l'une des catégories de Watts (1981), appelée toutefois différemment par ce dernier («applications de recherche»). Elle comprend trois catégories, soit l'acquisition de données (systèmes de collecte en temps réel), l'analyse de données (ensembles statistiques) et la modélisation (simulateurs, générateurs de solutions). La troisième catégorie (Gestion de l'information) regroupe les applications administratives habituelles.

Un deuxième sous-groupe de typologies centrées sur l'école s'appuie sur une classification des applications des TIC selon les divers acteurs d'une école. Deux typologies sont de ce sous-groupe. Dubuc (1982) répertorie les utilisations de l'informatique au sein d'une école pour chacun des trois principaux acteurs du système d'éducation : l'administrateur, le professeur et l'étudiant. Fait à noter, les exerciseurs et les tutoriels sont classifiés comme des instruments utilisés par l'enseignant et non par l'apprenant, probablement parce qu'à l'époque, on présumait encore que l'enseignant ferait le travail de conception de ces logiciels. En fait, la seule utilisation attribuée à l'apprenant est celle de l'ordinateur «automate », qui correspond à ce que Taylor (1980) appelle l'ordinateur-apprenant.

Collis (1996) distingue les usages de l'ordinateur orientés apprenant et les usages orientés enseignant. Les premiers sont subdivisés en trois catégories, soit les usages qui se font dans le cadre de tâches d'apprentissage structurées et 
prédéterminées (exerciseurs, tutoriels, jeux éducatifs, certaines simulations, certains logiciels de résolution de problèmes, culture informatique), ceux qui offrent une certaine flexibilité d'usage à l'apprenant à l'intérieur d'une tâche d'apprentissage ou d'un contenu prédéfinis (certaines simulations dont quelques micromondes, certains logiciels de résolution de problème, les laboratoires assistés par ordinateur, les banques de données; les hypertextes, certains systèmes experts et les sites web) et les outils ou environnements sans contenu (content-free) tels que les traitements de texte, les bases de données, les tableurs, les environnements de programmation, les environnements de télécommunication, de production visuelle et audio et de manipulation et les « outils cognitifs » (organisateurs d'idées, logiciels de création de cartes conceptuelles, etc.). Les usages orientés enseignant incluent les utilisations par l'enseignant d'outils informatiques pour l'acquisition et la manipulation de ressources et pour la communication. Ceci inclut tous les logiciels déjà mentionnés pouvant être utilisés par l'apprenant ainsi que les outils de production de matériel didactique et d'évaluation des apprentissages et enfin les outils de gestion de l'enseignement.

\subsection{Les typologies centrées sur l'apprenant}

Ce n'est que dans les années 1990 que ces typologies ont commencé à émerger, sans doute inspirées par les courants cognitivistes et constructivistes de l'apprentissage. Il s'agit de typologies qui font référence aux impulsions naturelles de l'apprenant, aux fonctions cognitives soutenues par les technologies, aux étapes du processus d'apprentissage ou encore aux étapes du processus humain de traitement de l'information telles que les ont définies les chercheurs en psychologie cognitive.

Bruce et Levin (1997) classifient les technologies éducatives selon les quatre impulsions naturelles de l'individu à apprendre telles que définies par Dewey (1943) : exploration (inquiry), communication, construction et expression. Les deux premières catégories nécessitent une spécification de catégories de deuxième niveau, compte tenu de la variété des technologies pouvant y être associées. Par exemple, les technologies d'exploration sont classifiées selon qu'elles servent à construire des théories (ex. : logiciels de visualisation, environnements de réalité virtuelle, etc.), à accéder à des données (ex. : bases de données, hypertexte, etc.), à recueillir des données (ex. : laboratoires informatisés, enregistrement sonore et vidéo, etc.) et à analyser des données (ex. : tableurs, analyse statistique, etc.).

Les fonctions cognitives pouvant être étendues ou restructurées par les TIC servent de critère de classification pour un deuxième sous-groupe de typologies centrées sur l'apprenant. C'est le cas de la typologie de Jonassen (2000), qui se rapproche toutefois fortement de celle de Bruce et Levin (1997). Comme dans sa typologie de 1995, Jonassen passe rapidement sur les applications regroupées habituellement sous le vocable «enseignement assisté par ordinateur », la culture 
informatique (computer literacy) et les outils de productivité comme le traitement de texte, pour porter ensuite toute son attention sur ce qu'il appelle des outils cognitifs (mindtools) : «Mindtools are computer-based tools and learning environments that have been adapted or developed to function as intellectual partners with the learner in order to engage and facilitate critical thinking and higher order learning » (p. 9). Les outils cognitifs ne font pas que faciliter l'exécution d'une tâche, comme le permet, par exemple, un traitement de texte. Ils restructurent et amplifient significativement la pensée de l'apprenant, en lui offrant la possibilité de représenter le savoir en utilisant de nouveaux langages formels. Ces outils sont regroupés en cinq catégories principales (Organisation sémantique, Modélisation dynamique, Interprétation, Construction du savoir et Conversation) et en plusieurs souscatégories, auxquelles sont associés des groupes plus spécifiques de technologies et des instanciations d'outils actuellement offerts sur le marché.

Chacón (1992) classifie les «médias informatiques » selon ce qu'il appelle les «modes utilisateur» (user-modes). Un mode utilisateur est une utilisation qu'un usager fait de l'ordinateur pour étendre ses fonctions humaines. Chacón en identifie trois principaux et associe, à chacun, une métaphore : le traitement de l'information (métaphore: «J'apprends en agissant»), l'interaction entre l'apprenant et la machine (métaphore: «Je m'enseigne quelque chose à moi-même») et la communication (métaphore: «J'apprends au moyen des autres»). À ces modes utilisateur, Chacón associe un certain nombre de technologies, qu'il situe également sur un continuum temporel. Par exemple, pour le mode utilisateur «communication», les technologies identifiées par Chacòn vont des banques interactives d'informations à la communication multimédia, en passant par le courrier électronique et les conférences télématiques. Cette perspective évolutive permet de rajouter, pour chacun des modes utilisateur, les nouvelles technologies qui sont introduites en éducation au fur et à mesure de leur développement.

Certaines typologies introduisent une dimension séquentielle dans les usages qu'un apprenant peut faire des TIC au cours de son processus d'apprentissage, formant ainsi un troisième sous-groupe de typologies centrées sur l'apprenant. Déjà en 1984, Thomas et Boysen proposaient une typologie fondée sur ce qu'ils considèrent les étapes du processus d'apprentissage: Expérimenter, Informer, Renforcer, Intégrer et Utiliser. Un ou deux exemples de technologies pouvant supporter ces étapes sont identifiés pour chacune. Quant à Iiyoshi et Hannafin (1998), ils s'appuient sur les théories classiques du traitement humain de l'information pour associer à chaque étape de ce processus une certaine classe d'outils informatiques susceptibles de la supporter. Ainsi, pour rechercher l'information, l'apprenant peut avoir recours à des outils de recherche d'informations (information seeking tools); pour sélectionner l'information, il peut recourir à des outils de présentation d'information (information presentation tools). Lorsque l'information se trouve dans la mémoire à court terme, il peut utiliser des outils d'organisation et d'intégration des informatios. Enfin, l'apprenant peut utiliser des outils de production lorsqu'il s'agit de récupérer des informations de la mémoire 
à long terme et de générer des connaissances. Les titres utilisés pour nommer ces classes se rapprochent beaucoup de certaines classes proposées par Jonassen (2000).

\section{Conclusion}

Selon Seels (1997), tout comme on favorise aujourd'hui des approches multiples de classification pour enrichir la discipline de la biologie au plan théorique comme au plan pratique, le champ de la technologie éducative a tout intérêt à multiplier les approches à la classification de divers phénomènes reliés au domaine.

L'analyse montre que cela est le cas pour ce qui est des typologies proposées par divers auteurs depuis le début des années 1980 pour classifier les applications des TIC en éducation. En effet, une variété de critères ont été utilisés pour ce faire. Certains sont reliés à différentes variables de l'acte d'enseignement/apprentissage (rôle pédagogique de l'ordinateur, degré d'autonomie de l'apprenant, type de stratégies pédagogiques, étapes du processus d'enseignement); d'autres sont associés aux types d'activités se déroulant dans un établissement d'enseignement ou aux acteurs pouvant bénéficier de leur usage, et d'autres enfin sont regroupés selon différentes caractéristiques cognitives d'un apprenant (ses impulsions à apprendre, ses fonctions cognitives, les étapes du processus d'apprentissage ou du traitement de l'information).

On constate que, dans la foulée du cognitivisme et du constructivisme, le fonctionnement cognitif de l'apprenant devient de plus en plus, à partir des années 1990, un pôle important des efforts de classification. Ces analyses conduisent à des typologies de plus en plus raffinées sur le rôle majeur que les TIC peuvent jouer non seulement comme soutien aux fonctions cognitives humaines mais également comme amplificateurs et comme source de restructuration de différentes activités cognitives (Jonassen, 2000).

On remarque, par ailleurs, que les typologies centrées sur l'acte d'enseignementapprentissage tendent à offrir une vision de plus en plus intégrée des différentes variables en interaction dans les situations pédagogiques intégrant les TIC: celles-ci sont associées non seulement à des activités pédagogiques qu'elles permettent de mettre en oeuvre mais également aux fonctions pédagogiques qu'elles peuvent assumer, aux objectifs d'apprentissage qu'elles sont susceptibles de favoriser et aux théories d'apprentissage avec lesquelles elles s'accordent davantage. La typologie de De Vries (2001) en est un bon exemple.

Les vingt-neuf typologies recensées offrent autant de regards différents posés sur le phénomène des TIC en éducation. Tant les théoriciens que les chercheurs et les praticiens en éducation gagnent à connaître cette variété de points de vue, à en cerner les similitudes, les complémentarités et les divergences, afin de développer un vocabulaire commun et d'enrichir leur propre carte conceptuelle du domaine. La 
typologie des typologies des usages des TIC en éducation présentée ici constitue un premier effort dans ce sens.

Il est difficile d'imaginer quelles technologies émergeront dans le futur et de prédire, comme le souligne Baron (1997), les nouvelles applications des TIC qui seront inventées par les développeurs, les chercheurs et les praticiens du domaine au cours des prochaines années. Il sera intéressant de suivre cette évolution et de vérifier si, conjuguée à l'évolution du domaine des sciences de l'éducation, de nouvelles typologies des applications des TIC en éducation en émergeront.

Pour l'heure, nous entrevoyons deux champs d'application où les typologies déjà existantes pourraient jouer un rôle profitable.

Premièrement, elles pourraient alimenter le développement d'ontologies dans le domaine de l'éducation, de la technologie éducative ou du design pédagogique (Bourdeau et Mizoguchi, 2002). En effet, divers chercheurs en intelligence artificielle développent des ontologies dans le but de représenter divers domaines de savoir (Gruber, 1993 ; Staab et al., 2001). Pour un domaine donné, il s'agit de spécifier une ou plusieurs taxonomies regroupant des concepts ordonnés hiérarchiquement, ainsi que leurs interrelations et des règles d'inférence permettant de définir des propriétés de ces relations. Ces chercheurs visent à élaborer un vocabulaire partagé par une communauté afin de permettre l'échange entre des agents informatiques au moment d'effectuer des recherches d'informations dans le web sémantique (Berners-Lee et al., 2001).

Deuxièmement, les typologies pourraient contribuer à résoudre en partie l'épineux problème de l'indexation de ce que l'on appelle les «objets d'apprentissage » (ou objets pédagogiques). ${ }^{5}$ Depuis quelques années, en effet, de nombreux travaux visant le développement de banques de tels objets pour le l'enseignement et l'apprentissage ont été amorcés (Wiley, 2001, 2002). Comme le souligne Bourda (2001), l'idée est d'aboutir à des composants pédagogiques réutilisables dans un but de mutualisation de documents pédagogiques ou de rentabilisation des productions pédagogiques. Or, le problème d'indexation de ces objets au moyen de ce que l'on appelle des métadonnées est loin d'être résolu (Bourda, 2001; Schatz, 2002). Les métadonnées permettent de référencer un objet de sorte qu'il soit repérable par des agents humains et informatiques. Plusieurs initiatives pour standardiser ce type de données sont entamées dans le monde entier (IMS, IEEE, ADL, etc.).

Pour que les objets d'apprentissage acquièrent une véritable valeur pédagogique, il faut aller au-delà de descripteurs purement objectifs (auteur, titre, langue, format, etc.) afin de les décrire (Bourda, 2001). Il faut leur attribuer des descripteurs révélant à la fois leurs contextes d'utilisation, leur structure et leur contenu. Pour ce

5 Wiley (2001) définit un objet d'apprentissage comme une entité digitale ou non, qui peut être utilisée, réutilisée ou référencée au cours de l'apprentissage assisté par les technologies. 
type de métadonnées, il a été suggéré d'utiliser, par exemple, la classification Dewey. Plusieurs projets, tels que MERLOT ${ }^{6}$, le gestionnaire de ressource Explor@2, CAREO, etc. (Friesen, 2002), proposent des classifications plus près de la pratique éducative. Ces classifications se veulent évolutives. Les typologies des applications des TIC en éducation pourraient ici être d'une grande utilité.

\section{Bibliographie}

Alessi, S. M., et Trollip, S. R., Computer-based instruction : Methods and development, Englewood Cliffs, Prentice-Hall, $2^{\mathrm{e}}$ édition, 1991.

Aylwin, U., «Les utilisations de l'informatique dans un établissement scolaire», Prospectives, février-avril-octobre, 1984, p. 13-17.

Baron, G.-L., «Des technologies "nouvelles" en éducation?», Recherche et Formation, $\mathrm{n}^{\mathrm{o}} 26,1997$, p. 121-130.

Baron, G.-L., Bruillard, É., L'informatique et ses usagers dans l'éducation, Paris, Presses universitaires de France, 1996.

Basque, J., Rocheleau, J., Winer, L., Michaud, P., Bergeron, G., Paquette, G., Paquin, C., Un modèle adaptable d'une école informatisée, Montréal, École informatisée clés en main du Québec inc., 1998, disponible à l'adresse http://www.grics.qc.ca/cles_en_main [consulté le 12 novembre 2002].

Baumgartner, P., Payr, S., «Learning with the Internet: A typology of application», Proceedings of ED-MEDIA/ED-TELECOM 98 (World Conference on Educational Multimedia and Hypermedia \& World Conference on Educational Telecommunications), Charlotesville, AACE, 1998, p. 124-129.

Berners-Lee, T., Hendler, J., Lassila, O., « The semantic web », Scientific American, vol. 284, $\mathrm{n}^{\mathrm{o}}$ 5, 2001, p. 35-43.

Bork, A., Personal Computers for Education, New York, Harper \& Row, 1985.

Bourda, Y., «Objets pédagogiques, vous avez dit objets pédagogiques? », Cahiers GUTenberg, $\mathrm{n}^{\mathrm{o}}$ 39-40, 2001, p. 71-79.

Bourdeau, J. et Mizoguchi, R., «Collaborative Ontological Engineering of Instructional Design Knowledge for an ITS Authoring Environment », dans S. Cerri,, G. Gouardères, F. Paraguaçu, Intelligent Tutoring Systems, Lecture Notes in Computer Science, Heidelberg, Springer, 2002, p. 399-409.

Bruce, B. C., Levin, J. A., «Educational technology : Media for inquiry, communication, construction and expression », Journal of Educational Computing Research, vol. 17, $\mathrm{n}^{\mathrm{o}} 1,1987$, p. $79-102$.

\footnotetext{
${ }^{6}$ MERLOT : http://www.merlot.org/Home.po [consulté le 12 novembre 2002].
} 
Chacón, F., « A taxonomy of computer media in distance education », Open Learning, vol.. $7, \mathrm{n}^{\circ} 1,1992$, p. 12-27.

Collis, B. A., «Computers in education», dans T. Plomp, D. P. Ely (éds.), International encyclopedia of educational technology, Oxford, Elsevier Science, 1996, $2^{\mathrm{e}}$ édition, p. 402-408.

de Vries, E., «Les logiciels d'apprentissage: panoplie ou éventail?», Revue Française de Pédagogie, ${ }^{\circ}$ 137, p. 105-116.

Denis, B., Quels usages des logiciels mettre en oeuvre en contexte éducatif? http://www.agers.cfwb.be/pedag/tice/sugg/Article\%20UPTICE1.pdf2 [consulté le 12 novembre 2002].

Dewey, J., The child and the curriculum / The school and society, Chicago, University of Chicago Press, 1943.

Dubuc, L., Classification des applications pédagogiques de l'ordinateur, Montréal, Direction générale des moyens d'enseignement, Ministère de l'Éducation du Québec, 1982.

Forcier, R. C., The computer as an educational tool: Productivity and problem solving, Upper Saddle River, Prentice-Hall, 1999, $2^{\mathrm{e}}$ édition, 1999.

Friesen, N., Roberts, A., Fisher, S., CanCore: Learning Object Metadata, 2002, http://www.cancore.ca/cancorepaper.doc [consulté le 12 novembre 2002].

Gruber T. R., Toward Principles for the Design of Ontologies Used for Knowledge Sharing, Technical Report KSL-93-04, Knowledge Systems Laboratory, Stanford University, 1993, http://citeseer.nj.nec.com/cachedpage/100962/1 [consulté le 12 novembre 2002].

Harris, J., «Organizing and Facilitating Telecollaborative Projects», The Computing Teacher, vol. 22, no 5, 1995, p. 66-69.

Heinich, R., Molenda, M., Russel, J. D., Instructional media and the new technologies of instruction, New York, Macmillan, $4^{\mathrm{e}}$ édition, 1993.

Iiyoshi, T., Hannafin, M. J., «Cognitive tools for open-ended learning environments : Theoretical and implementation perspectives », Communication AERA (American Educational Research Association), San Diego, 13-17 avril 1998.

Jonassen, D. H., «Supporting communities of learners with technology: A vision for integrating technology with learning in schools », Educational Technology, vol. 35, nº 4, 1995, p. 60-63.

Jonassen, D. H., Computers as mindtools for schools : Engaging critical thinking, Upper Saddle River, Prentice Hall, $2^{\mathrm{e}}$ édition, 2000.

Knezek, G., Rachlin, S. L., Scannell, P., «A Taxonomy for Educational Computing », Educational Technology, vol. XXVIII, n ${ }^{\circ} 3,1988$, p. 15-19.

Lebrun, M., Des technologies pour enseigner et apprendre, Bruxelles, De Boeck, 2 édition, 2002.

Legendre, R., Dictionnaire actuel de l'éducation (2e éd.), Montréal/Paris, Guérin/Eska, $2^{\mathrm{e}}$ édition, 1993. 
26 Sciences et techniques éducatives. Volume $9-\mathrm{n}^{\circ}$ 3-4/2002, pages 263-298.

Limbos, B., «Essai de classification des utilisations pédagogiques de l'ordinateur selon cinq situations d'apprentissage », Dossiers pédagogiques no 5, Fédération de l'enseignement $\begin{array}{lll}\text { fondamental catholique, } & \text { Bruxelles, }\end{array}$ www.segec.be/fededoc/publications/juin/classification.pdf [consulté le 2 novembre 2002].

Means, B., «Introduction : Using Technology to Advance Educational Goals », dans B. Means (éd.), Technology and Education reform: The Reality Behind the Promise, San Francisco, Jossey-Bass, 1994, p. 1-21.

Ministère de la Jeunesse, de l'Éducation nationale et de la Recherche, « Typologie des usages pédagogiques des TIC pour les élèves et les enseignants », www.educnet.education.fr/primaire/typologie, htm [consulté le 2 novembre 2002 ].

Paquette, G., «Les logiciels de formation», dans G. Paquette (éd.), Initiation à la formation/conseil en milieu de travail, Sainte-Foy (Québec), Télé-université, 1993, p. 299-315.

Plante, J.-L., « Une classification ouverte des application pédagogiques de l'ordinateur », Vie pédagogique, $\mathrm{n}^{\mathrm{0}} 31,1984$, p. 26-29.

Porter, D., Curry, J., Muirhead, B., Galan, N., Les référentiels d'objets d'apprentissage, CANARIE et Industrie Canada, 2002.

Pressey, S. L., «A simple device which gives tests and scores - and teaches » ». School and Society, vol. 23, 1926, p. 373-376.

Reiser, R. A., «A history of instructional design and technology. Part I: A history of instructional media», Educational Technology Research \& Development, vol. 49, no 1, 2001, p. 53-64.

Roecks, A. L., « How many ways can the computer be used in education? A baker's dozen », Educational Technology, vol. 21, $\mathrm{n}^{\circ}$ 9, 1981, p. 16.

Romiszowski, A. J., Designing instructional systems. Decision making in course planning and curriculum design, London/New York, Kogan Page/Nichols Publishing, 1992.

Sauvé, L., Document du cours INF 6001 Ordinateur et environnement éducatif, Montréal, Québec, Télé-université, 1984, p. 28-34.

Schatz, S., Paradigm Shifts and Challenges for Instructional Designers. An Introduction to Meta Tags and Knowledge Bits, http://citeseer.nj.nec.com/correct/531084 [consulté le 2 novembre 2002 ].

Schultz, K. A., Hart, L. C., «Computers in education », Arithmetic Teacher, vol. 31, $\mathrm{n}^{\circ} 4$, 1983, p. 36-37.

Seels, B., «Taxonomic issues and the development of theory in instructional technology » Educational Technology, vol. 37, $\mathrm{n}^{\mathrm{o}}$ 1, 1987, p. 12-21.

Séguin, P., Internet, une technologie pour l'apprentissage, Montréal, Collège Bois-deBoulogne, 14 septembre 1997, http://www.colvir.net/pedagogie/ [consulté le 12 novembre 2002]. 
Skinner, B. F., «The science of learning and the art of teaching ", Harvard Educational Review, vol. 24, n 2, 1954, p. 86-97.

Staab, S., Studer, R., Schnurr, H.P., Sure, Y., « Knowledge processes and ontologies », IEEE Intelligent Systems, vol. 16, $\mathrm{n}^{\mathrm{o}} 1$, 2001, p. 26-34.

Taylor, R. P., The Computer in the School: Tutor, Tool, Tutee, New York, Teachers College Press, 1980.

Thomas, R. A., Boysen, J. P, « A taxonomy for the instructional use of computers », AEDS Monitor, vol. 22, $\mathrm{n}^{\circ} 11-12,1984$, p. 15-17.

Watts, N., «A dozen uses for the computer in education », Educational Technology, vol. 21, $\mathrm{n}^{\circ} 4,1981$, p. 18-22.

Wiley, D. A., «Connecting learning objects to instructional design theory: A definition, a metaphor, and a taxonomy », dans D.A. Wiley (éd.), The instructional use of learning objects, Bloomington, Indiana, Association for Educational Communications of Technology, 2001

Wiley, D. A., Edwards, E. K., «Online self-organizing social systems: The decentralized future of online learning ", Quarterly Review of Distance Education, accepté 2002, http://wiley.ed.usu.edu/docs/ososs.pdf [consulté le 12 novembre 2002 ].

Josianne Basque détient un doctorat en Psychologie de l'Université du Québec à Montréal. Elle est professeure à la Télé-université en technologies appliquées à l'éducation depuis 1997. Elle est également chercheure au Centre de recherche LICEF (Laboratoire en informatique cognitive et environnements de formation) de la Télé-université et au Centre interuniversitaire de recherche sur le téléapprentissage (CIRTA). Dans ses recherches, elle s'intéresse à la co-construction de connaissances à l'aide des TIC, à la métacognition et à l'ingénierie pédagogique. Avant de devenir professeure, elle a réalisé plusieurs études et recherches dans le domaine des TIC appliquées à l'éducation, notamment au ministère de l'éducation du Québec, au Centre québécois de recherche sur les applications pédagogiques de l'ordinateur (APO QUÉBEC) et au Centre de recherche LICEF.

Karin Lundgren-Cayrol détient un doctorat en Technologie de l'éducation de l'Université Concordia. Depuis six ans, elle travaille comme chercheure associée au Centre de recherche LICEF de la Télé-université. Elle est co-auteure, avec France Henri, de l'ouvrage "Apprentissage collaboratif à distance: Pour comprendre et concevoir les environnements d'apprentissage virtuel » publié aux Presses de l'Université du Québec. Ses intérêts de recherche portent sur les stratégies pédagogiques et les outils informatiques pour faciliter l'apprentissage virtuel. 